\title{
MANEJ O DE LA INFECCIÓN POR CANDIDA EN EL RECIÉN NACIDO
}

\author{
Diana A rias F. M D*, J uan Carlos J iménez S.M D**
}

\section{Resumen}

A nivel mundial la mortalidad neonatal corresponde al $41 \%$ de las que ocurren en menores de cinco años. Dentro de las causas descritas por la OMS la sepsis corresponde a la tercera, después de la prematurez y la asfixia perinatal. La Candida sp es uno de los agentes etiológicos que en el período neonatal genera alta morbilidad y mortalidad, así como secuelas neurológicas a largo plazo en especial en prematuros y de bajo peso. Estos pacientes cuentan con características inmunológicas propias, requerimientos de procedimientos invasivos y mayor sobrevida en estancias hospitalarias prolongadas, que se convierten en factores de riesgo que predisponen a desarrollar candidiasis sistémica. Dado el impacto de esta patología es importante contar con un abordaje integral diagnóstico y terapéutico eficaz que disminuya la morbimortalidad, mejorando el pronóstico vital y funcional. En los últimos años se han generado diferentes guías y protocolos médicos en busca de optimizar el manejo de esta infección, por lo que es importante describir cuales son las mejores recomendaciones actuales para el enfoque, diagnóstico y tratamiento de la candidiasis neonatal.

Palabras clave: $\mathbf{R N}$, recién nacido(s).

\section{MANAGEMENT OF CANDIDA INFECTION}

\begin{abstract}
Worldwide, neonatal mortality accounts for $41 \%$ of deaths occurring in children younger than 5 years. Among the causes described by the WHO, sepsis corresponds to the third most common cause of death, after prematurity and perinatal asphyxia. Candida sp is one of the etiologic agents which generate high morbidity and mortality, as well as, long-term neurologic sequelae predominantly in low birth-weight preterm infants. These patients have particular immunological features, require invasive procedures and greater survival in prolonged hospital stays, which become predisposing risk factors for the development of systemic candidiasis. Due to the impact of this condition it is important to count with a comprehensive diagnostic approach which reduces morbidity and mortality improving vital and functional prognosis. Various medical guidelines and protocols seeking candida infection management optimization have been recently developed, thus, it is essential to describe the best current recommendations to address, diagnose and treat neonatal candidiasis.
\end{abstract}

Key words: NB, newborn(s).

Fecha recibido: abril 4 de 2014 - Fecha aceptado: marzo 15 de 2015

* Médica pediatra, neonatóloga. Jefe del Servicio de Pediatría Hospital de San José. Instructor Asistente, Fundación Universitaria de Ciencias de la Salud. Bogotá DC, Colombia.
** Residente III de Pediatría, Fundación Universitaria de Ciencias de la Salud. Bogotá DC, Colombia. 


\section{Introduc ción}

L a Candida es la micosis más común en neonatos que abarca desde infecciones leves hasta formas graves, con elevada mortalidad en especial en pretérminos menores de $1.000 \mathrm{~g} .{ }^{1,2} \mathrm{H}$ a tomado fuerza por el advenimiento de mejor tecnología en el cuidado de RN de bajo peso. Se estima que afecta a $1 \%$ de los R N entre 1.000 y $1.500 \mathrm{~g}$ y cerca de $12 \%$ a menores de 750 $g$, lo cual aumenta la mortalidad en las unidades de cuidado neonatal hasta $73 \%$ en menores de $1.000 \mathrm{~g}$, así como el deterioro del neurodesarrollo. ${ }^{3} \mathrm{~L}$ a mayoría de las infecciones de trasmisión vertical son generadas por C. albicans y las tipo horizontal por C. parapsilosis. ${ }^{4,5}$ Los medicamentos usados para el manejo de las infecciones invasoras por hongos en $\mathrm{RN}$ se han agrupado en cuatro categorías, con base en las experiencias de los estudios en adultos y la evidencia en neonatos. A unque se saben los mecanismos de acción y las reacciones adversas, los estudios en este grupo de edad son limitados. ${ }^{6,7}$ Con base en lo anterior se deduce la importancia de establecer guías para el manejo de esta infección, formulando pautas de manejo en la población de mayor riesgo y situaciones puntuales derivadas, así como las estrategias para la prevención en indicaciones de profilaxis.

\section{Definición}

L a candidemia es definida por un cultivo positivo en un líquido que debe ser estéril. En los neonatos el principal foco es el torrente sanguíneo (cerca del $70 \%$ de los casos), otros sitios de aislamiento son el tracto urinario (15\% por cateterismo vesical o punción suprapúbica) y el líquido cefalorraquídeo $(10 \%){ }^{8}$

\section{Epidemiología}

La mayoría de las infecciones son generadas por $C$. albicans y $C$. parapsilosis, aunque también por otras especies de $C$ andida, siendo más común la trasmisión vertical para $\mathrm{C}$. albicans y horizontal para $\mathrm{C}$. parapsilosis. ${ }^{9,10} \mathrm{~L}$ a mayor población está constituida por recién nacidos pretérmino en especial con peso inferior a $1.000 \mathrm{~g}$, debido a la inmadurez dada en especial por defectos en la quimiotaxis, fagocitosis y producción de anticuerpos. ${ }^{11,12}$ Esto ha determinado que la incidencia de la enfermedad sea inversamente proporcional al peso del neonato (Tabla 1)..$^{11-13}$

Es importante aclarar que la aparición de la infección está relacionada con otros factores que determinan el pronóstico y la sobrevida de los pacientes, como el tiempo de estancia en la unidad, comorbilidades, el uso de dispositivos invasivos y de antibióticos de amplio espectro, los cuales alteran la microflora y generan competencia entre gérmenes gram negativos y anaerobios, favoreciendo además la invasión por hongos y otros microorganismos, todo ello influenciado también por los índices de colonización que tengan las diferentes unidades de cuidado neonatal, índice que puede variar según la complejidad de cada institución (Tabla 2). ${ }^{14,15}$

\begin{tabular}{|l|c|}
$\begin{array}{c}\text { Tabla I. Incidencia de candidiasis en recién } \\
\text { nacidos según peso }\end{array}$ \\
\hline Peso en gramos & $\begin{array}{c}\text { Incidencia de infección por } \\
\text { Candida }\end{array}$ \\
\hline Mayores de 1.500 & $1 \%$ \\
\hline $1.001-1.499$ & $1 \%$ \\
\hline $751-1.000$ & $4 \%$ \\
\hline $450-700$ & $12 \%$ \\
\hline
\end{tabular}

\begin{tabular}{|l|l|}
\multicolumn{1}{|c|}{ Tabla 2. Factores de riesgo asociados con } \\
Candidiasis invasiva
\end{tabular}

*Inhibidor de la bomba de protones, CVC = catéter venoso central, CID = coagulación intravascular diseminada. 
Por lo regular la infección sistémica por candida se presenta luego de la segunda semana de estancia; en el $80 \%$ de los casos se diagnostica en los primeros 42 días de edad de pretérminos extremos, lo que impacta la mortalidad pues el $40 \%$ de pacientes de al menos 750 g morirá, comparado con $20 \%$ de pretérminos entre 1.000 y $1.500 \mathrm{~g}^{16,17}$

\section{Aspectos terapéuticos:}

\section{¿Qué medicamentos se usan para la infección fúngica?}

Los que se han empleado para el manejo de las infecciones invasoras por hongos en $\mathrm{R} N$ se agrupan en cuatro categorías basadas en la experiencia de los estudios en adultos y la evidencia del empleo en neonatos, que si bien conocemos los mecanismos de acción y las reacciones adversas en este grupo de edad, los estudios son limitados ${ }^{18}$ (Tablas 3 y 4).

Polienos: Ia anfotericina B se ha usado desde 1958 en adultos, después se extrapoló su uso a los niños en donde se ha encontrado mayor penetrabilidad en el sistema nervioso central y mejor actividad en fungemias invasoras. ${ }^{19}$ Su acción se basa en el bloqueo de la unión del ergosterol en la membrana citoplasmática del hongo, dando como resultado formación de porosidades en esta y actuando como fungicida. Tiene baja absorción por vía oral y la capacidad de unión a las proteínas es cerca del $95 \%$ con un amplio volumen de distribución. ${ }^{19}$

D eoxicolato: es la forma más conocida, con las características antes mencionadas de la anfotericina. En los niños tiene capacidad de concentración en hígado, bazo y riñones, así como una vida media más larga que en los adultos (15 horas). El principal problema de esta

\begin{tabular}{|c|c|c|c|}
\hline Polienos & Triazoles & Equinocandinas & $\begin{array}{c}\text { Análogo } \\
\text { nucleósido }\end{array}$ \\
\hline $\begin{array}{l}\text { Anfotericina B } \\
\text { AmB-d* } \\
\text { L-AmB** } \\
\text { ABLC*** } \\
\text { ABCD**** }\end{array}$ & $\begin{array}{l}\text { Fluconazol } \\
\text { Voriconazol } \\
\text { Itraconazol } \\
\text { Pasaconazol }\end{array}$ & $\begin{array}{l}\text { Caspofungina } \\
\text { Anidulafungina } \\
\text { Micafungina }\end{array}$ & Flucitosina \\
\hline
\end{tabular}

*Deoxicolato, ** liposomal, *** complejo lipídico, ****dispersión coloidal. forma terapéutica es la capacidad de daño renal que se ha visto hasta en $16 \%$ de pacientes entre 26 y 41 semanas, acompañado de hipocalemia en $17 \%,{ }^{20}$

Formas lipídicas: se han desarrollado tres formas de anfotericina en las cuales se han visto menores efectos adversos que en la tradicional, sin embargo el uso en niños aún se restringe. $L$ a forma liposomal ( $L$-amB) se aprobó en mayores de un mes, pero hay revisiones en las cuales se ha usado con éxito en el $\mathrm{R} N$ de bajo y muy bajo peso al nacer sin mayores efectos adversos que la forma deoxicolato. Están también el complejo lipídico $(A B L C)$ que se aprobó para mayores de 16 meses y la anfotericina de dispersión coloidal ( $A B C D$ ) aplicable solo en niños mayores y adultos. ${ }^{19}$ A unque se ha establecido la toxicidad por anfotericina $B$ en cuanto a los efectos renales, no se ha demostrado en RN que la forma deoxicolato sea más toxica que las lipídicas, pero en pacientes mayores no ocurre de la misma manera, razón por lo cual no se aconseja. ${ }^{21}$

Azoles: estos medicamentos interfieren con el metabolismo del ergosterol inhibiendo la lanoesterol 14-a-demetilasa, que hace parte de la P450 y de esta manera inhibe la conversión de lanosterol a ergosterol, alterando así la membrana celular del hongo. Los principales representantes de este grupo terapéuticos son fluconazol, voriconazol e itraconazol, los cuales son activos contra varias especies de Candida y en menor proporción a C. Krusei y C, G labrata., ${ }^{22,7}$ El fluconazol es uno de los más usados en infección y aunque la recomendación en el manejo de la candidiasis invasora es la anfotericina, se plantea como una opción teniendo en cuenta la buena penetrabilidad en los tejidos así como en el sistema nervioso central y en el tracto urinario. A demás ha demostrado ser seguro inclusive en RN pretérmino de muy bajo peso, con mínimos efectos a nivel hepatobiliar y gastrointestinal como reacciones adversas más comunes. El voriconazol por su parte tiene un espectro más amplio pero posee mayor acumulación una vez se ha establecido el daño renal, sumado a compromiso retiniano en pretérminos por lo que no se recomienda de rutina en la candidiasis del RN.7,23

Equiniocandinas: son un grupo reciente para el tratamiento de infecciones fúngicas con buenos resultados 
de seguridad y eficacia en el manejo de candidiasis invasora. Representados en la caspofungina, anidulafungina y micafungina inhiben la 1,3-B-D-glucan sintasa, enzima con acción en la síntesis de pared celular. La primera es una buena opción en los casos de candidiasis refractarias a tratamiento con anfotericina y fluconazol, son pocos los efectos adversos evidenciados en niños mayores, sin embargo por el poco uso en neonatos se ha establecido que se debe monitorear el estado electrolítico y la función hepática debido a el metabolismo de esta, pero no es necesario su ajuste en insuficiencia renal. ${ }^{24,25}$

Análogos nucleósidos: la flucitosina actúa bloqueando la síntesis de ADN y el ARN como metabolito del 5-Fluoracilo, sin embargo a pesar de tener buena actividad contra Candida y alcanzar buena distribución en los tejidos, tiene alto riesgo de resistencia como monoterapia, es por eso que se usa casi siempre como coadyuvante en el manejo con anfotericina. Su utilización es escasa en el RN, por los efectos adversos y las situaciones ya mencionadas. ${ }^{21,26}$

\section{¿Cuál es el tratamiento empírico en los recién nacidos con sospe- cha de Candida?}

Se ha definido la importancia del tratamiento adecuado una vez se sospecha la infección por $C$ andida en $\mathrm{RN}$ en especial los pretérmino de muy bajo peso al nacer. Una vez se demuestra presencia de levaduras en sangre, orina o líquido cefalorraquídeo, el tratamiento debe enfocarse a la erradicación de las mismas a fin de impactar en la mortalidad que genera y además de ello en los trastornos del neurodesarroIlo que se presentan en este tipo de pacientes. ${ }^{9,27,28}$ Son limitantes importantes la baja sensibilidad de los cultivos para detectar el microorganismo en el torrente sanguíneo que son positivos en $28 \%$ y la toma pequeña de 0.1 a $1 \mathrm{ml} .{ }^{28,8}$ Se recomienda el inicio de anfotericina $B$ deoxicolato a dosis de $1 \mathrm{mg} / \mathrm{k} /$ día en $R N$, en especial menores de $1.500 \mathrm{~g}$, en los cuales se presenten al menos seis de los nueve criterios que se mencionan en la (Tabla 5). ${ }^{10,8}$

RECOMENDACIÓN DEBIL, EVIDENCIA MODERADAIIB.

\begin{tabular}{|l|c|l|}
\multicolumn{3}{|c|}{$\begin{array}{c}\text { Tabla 4. Dosis de los principales antifúngicos } \\
\text { usados en recién nacidos }\end{array}$} \\
\hline \multicolumn{1}{|c|}{ Medicamento } & Ruta & \multicolumn{1}{c|}{ Dosis+ } \\
\hline Anfotetricina B Deoxicolato & IV & I mg/k/día \\
\hline Anfotetricina B Liposomal & IV & $5 \mathrm{mg} / \mathrm{k} /$ día \\
\hline Flucitosina & VO & $\begin{array}{l}50-150 \mathrm{mg} / \mathrm{k} / \text { dosis } \\
\text { cada } 6 \mathrm{horas}\end{array}$ \\
\hline Fluconazol & IV -VO & $12 \mathrm{mg} / \mathrm{k} / \mathrm{día}$ \\
\hline Caspofungina & IV & $25-50 \mathrm{mg} / \mathrm{m} 2 /$ día \\
\hline Anidulafungina & IV & $1.5 \mathrm{mg} / \mathrm{k} /$ día \\
\hline
\end{tabular}

+ Dosis en menores de 30 días.

Tabla 5. Criterios para iniciar terapia antifúngica para prevenir la candidiasis diseminada

\begin{tabular}{|l|l|}
\hline Inestabilidad hemodinámica & $\begin{array}{l}\text { Estancia en UCIN* con alta } \\
\text { incidencia del hongo }\end{array}$ \\
\hline $\begin{array}{l}\text { Uso prolongado de } \\
\text { antibióticos de amplio } \\
\text { espectro }\end{array}$ & $\begin{array}{l}\text { Uso de cefalosporinas de tercera } \\
\text { generación }\end{array}$ \\
\hline Ventilación mecánica & $\begin{array}{l}\text { Cultivos negativos persistentes a } \\
\text { pesar de signos clínicos de sepsis }\end{array}$ \\
\hline Exposición a esteroides & Trombocitopenia persistente \\
\hline $\begin{array}{l}\text { Recién nacidos sin } \\
\text { alimentación enteral. }\end{array}$ & \\
\hline
\end{tabular}

*UCIN: unidad de cuidado intensivo neonatal.

Los estudios clínicos han mostrado que el uso de anfotericina B como terapia dirigida contra Candida en $\mathrm{RN}$ ha disminuido la mortalidad y los eventos adversos en el neurodesarrollo, pero no se ha demostrado que el inicio precoz de la terapia con anfotericina o fluconazol impacte la mortalidad o los desenlaces asociados con la enfermedad. ${ }^{2}$ Se recomienda tratamiento por 14 días desde el momento en que se documenten cultivos negativos (Tablas 2 y 5). ${ }^{29,10,18}$

\section{¿Cuál debería ser el tratamiento según la especie de Candida aislada?}

Si se aisla C. albicans, C. parapsilosis o C. tropicalis se pueden utilizar anfotericina $\mathrm{B}$ o fluconazol. . $^{30,31}$

RECOMENDACIÓN FUERTE, EVIDENCIA ALTA- IA. 
A nte la presencia de C. G labrata: Ia anfotericina B es la primera elección. ${ }^{18}$

RECOMENDACIÓN DÉBIL, EVIDENCIA MODERADA - IIIB.

L a segunda opción de manejo es fluconazol en especial en los pacientes menos críticos. ${ }^{32,9}$

RECOMENDACIÓN DÉBIL, CALIDAD BAJA - IIIC.

Si es aislado C. krusei, tratar con anfotericina B..$^{33}$

RECOMENDACIÓN DÉBIL, CALIDAD BAJA - IIIC.

Para C. Iusitaniae se trata solo con fluconazol. ${ }^{9}$

RECOMENDACIÓN DÉBIL, EVIDENCIA MODERADA - IIIB.

\section{¿Cómo debería ser el tratamiento} en ciertas situaciones específicas de infección por Candida en recién nacidos?

\section{Candidemia:}

a) Se indica manejo prolongado de tres semanas después del último hemocultivo negativo., ${ }^{9,18}$

b) RECOMENDACIÓN DÉBIL, EVIDENCIA MODERA$D A-I I I B$.

c) La anfotericina B puede cambiarse por fluconazol para completar el tratamiento por vías parenteral u oral según el patrón de sensibilidad. ${ }^{34}$

d) RECOMENDACIÓN DÉBIL, EVIDENCIA MODERADA - IIIB.

e) Se deben retirar los catéteres intravasculares existentes en todos los casos de hemocultivos positivos para cualquier especie de Candida. 8,9 .

f) RECOMENDACIÓN FUERTE, EVIDENCIA ALTA-IA.

g) Realizar al menos una valoración por oftalmología para descartar endoftalmitis. 35
Candidiasis neonatal congénita diseminada:

h) Los recién nacidos pretérmino, en especial los de muy bajo peso o con ruptura prematura de membranas y cuadro clínico sugestivo, deben recibir manejo sistémico con anfotericina $B$ hasta completar $25 \mathrm{mg} / \mathrm{k}$ dosis acumulada o fluconazol como segunda elección. ${ }^{36}$

RECOMENDACIÓN DÉBIL, EVIDENCIA MODERADA - IIIB

En recién nacidos a término sin evidencia de candidemia se puede utilizar tratamiento tópico. ${ }^{26}$

\section{Candidiasis urinaria:}

1) La candiduria debe ser tratada en todos los sintomáticos y en neonatos de bajo peso. ${ }^{37}$

RECOMENDACIÓN DÉBIL, EVIDENCIA ALTA - IIIA.

2) El fluconazol es el medicamento de elección en los pacientes con infección de vías urinarias por C andida, siempre que haya estabilidad hemodinámica pues tiene una excelente penetrabilidad a la vía urinaria así como al SNC. ${ }^{38}$

3) La anfotericina B es la droga de elección en caso de inestabilidad hemodinámica. ${ }^{20}$

4) El tiempo de tratamiento se recomienda entre $7 y$ 14 días, siempre que no exista compromiso de otro sistema. ${ }^{22}$

RECOMENDACIÓN DÉBIL, EVIDENCIA ALTA - IIIA.

5) Se deben retirar los catéteres urinarios y no se indica la irrigación de la vejiga en forma regular, aunque puede ser útil en algunos casos de C. glabrata resistente a fluconazol. ${ }^{16}$

6) RECOMENDACIÓN DÉBIL, EVIDENCIA MODERADA- IIIB.

7) Se recomienda punción lumbar y evaluación del fondo de ojo en los casos de cultivos positivos en orina para cualquier especie de $\mathrm{C}$ andida. ${ }^{16}$

RECOMENDACIÓN DÉBIL, EVIDENCIA MODERADA-IIIB. 
8) En los casos de bolas fúngicas se considera el manejo quirúrgico como primera opción en $\mathrm{RN}$, así como el manejo con fluconazol en dosis de 12 $\mathrm{mg} / \mathrm{k} / \mathrm{dí}$, hasta que el cultivo sea negativo. ${ }^{39}$

RECOMENDACIÓN DÉBIL, EVIDENCIA MODERADAIIIB.

\section{Meningitis por Candida:}

a) Teniendo en cuenta la tendencia de esta enfermedad a las recaídas, el tratamiento debe administrarse por lo menos durante cuatro semanas después de la resolución de todos los signos y síntomas relacionados con la infección. La anfotericina B con flucitosina es la terapia inicial indicada. ${ }^{40}$

b) En caso de existir infección en SNC e inestabilidad hemodinámica, la elección es el uso de anfotericina $B$ deoxicolato en las primeras 48 a 72 horas; una vez se haya establecido la sensibilidad y las condiciones del paciente lo permitan se debe tratar de llevar a manejo con fluconazol. ${ }^{33}$

c) RECOMENDACIÓN DÉBIL, EVIDENCIA MODERA$D A-I I I B$.

d) Las válvulas de derivación ventrículoperitoneal deben ser retiradas. ${ }^{8}$

\section{Endocarditis por Candida:}

a) El manejo indicado es con anfotericina $B$ en dosis máxima durante seis semanas. ${ }^{10}$

b) RECOMENDACIÓN DÉBIL, EVIDENCIAALTA- IIIA.

Como se pueden presentar recaídas, se requiere seguimiento cuidadoso por lo menos durante un año. El tratamiento por largo tiempo con fluconazol se ha usado después del manejo inicial. En neonatos con bajo peso el manejo médico sin cirugía ha demostrado ser efectivo. ${ }^{6,41}$

\section{Endoftalmitis por Candida:}

a) M anejo inicial con anfotericina $B$ hasta completar la resolución completa o estabilización de la enfermedad por 6 a 12 semanas. $^{9}$

RECOMENDACIÓN DÉBIL, EVIDENCIA MODERADAIIIB. b) El fluconazol puede utilizarse para completar el manejo cuando es sensible. ${ }^{9}$

c) RECOMENDACIÓN DÉBIL, EVIDENCIA M ODERADA- IIIB.

d) Todos deben tener por lo menos una valoración por el oftalmólogo. ${ }^{9,35}$

e) RECOMENDACIÓN DÉBIL, EVIDENCIA M ODERA$D A-\| B$.

\section{Osteomielitis y artritis por Candida:}

a) Esta indicado el desbridamiento quirúrgico al igual que el manejo con anfotericina B por dos a tres semanas, seguido por fluconazol para un total de seis a doce meses. ${ }^{19}$

b) RECOMENDACIÓN DÉBIL, EVIDENCIA MODERADA- IIIB.

c) El uso de terapia intraarticular no está recomendada.

En los casos de los recién nacidos que tengan cultivos positivos a pesar del manejo, se deben realizar estudios de imágenes en busca de lesiones fúngicas en vías urinarias, bazo o hígado. ${ }^{10}$

RECOMENDACIÓN DÉBIL, EVIDENCIA MODERADAIIIB.

El uso de equinocandinas se restringe a las situaciones en las cuales la toxicidad renal no permite el uso de anfortercina B o fluconazol. ${ }^{25,29}$

RECOMENDACIÓN DÉBIL, EVIDENCIA MODERADAIIIB.

¿Está recomendado el uso de profilaxis para infección por Candida en todos los recién nacidos?

L as unidades de cuidado neonatal que tengan incidencia superior al $5 \%$ se benefician de profilaxis en los R N pretérminos por debajo de 1.000 gramos o menores de 28 semanas. ${ }^{42}$

RECOMENDACIÓN FUERTE, EVIDENCIA ALTA - IA. 
Hay suficiente evidencia para apoyar el uso de fluconazol con el objeto de disminuir la mortalidad debido a infección micótica sistémica. Sin embargo, está por establecerse el impacto a largo tiempo de esta intervención. 43,34 Se recomienda el uso de fluconazol a 3 $\mathrm{mg} / \mathrm{k}$ dos veces en la semana durante seis semanas. ${ }^{32,44}$

RECOMENDACIÓN FUERTE, EVIDENCIA ALTA - IA.

El uso intravenoso por seis semanas en prematuros menores de $1.000 \mathrm{~g}$ en dosis de $3 \mathrm{mg} / \mathrm{k} /$ día y aún más en aquellos sitios con alta incidencia de candidemia, puede disminuir la enfermedad invasiva. ${ }^{45} \mathrm{~N}$ o hay evidencia fuerte que apoye el uso de nistatina para la prevención de enfermedad micótica sistémica en las unidades de cuidado intensivo. ${ }^{46,32}$

\section{RECOMENDACIÓN DÉBIL, EVIDENCIA MODERADA-} IIIC.

\section{¿Qué estrategias pueden implemen- tarse para disminuir la infección por Candida en las unidades de recién nacidos?}

No hay evidencia fuerte que apoye el aislamiento para disminuir la infección cruzada por $C$ andida en unidades de recién nacidos; se han establecido al gunas otras medidas que apoyan el control de la infección. ${ }^{47,48}$

Manejo de líneas vasculares: se recomienda el uso de sistemas vasculares cerrados con adecuados antisépticos y apósitos transparentes alrededor de la implantación del catéter. Estos deben retirarse ante la sospecha de infección sistémica que requiera iniciar el tratamiento. Se ha demostrado que la evolución es mejor en aquellos a quienes se les retira el catéter en los primeros tres días cuando se identifican cultivos positivos. Se deben realizar cambios periódicos de accesos centrales aunque no se ha establecido cual debe ser el tiempo mínimo para realizarlos. ${ }^{49,50}$

U so racional de medicamentos: es indispensable el uso racional de antibióticos de amplio espectro, como cefalosporinas de tercera generación y carbapenémicos. Se deben restringir los anti $\mathrm{H} 2$ y el uso de dexametasona postnatal. ${ }^{16}$
Lactancia: se debe estimular la iniciación temprana de la lactancia materna como estrategia para disminuir la aparición de enterocolitis necrosante. ${ }^{39}$

\section{Referencias}

1. Smith PB, Steinbach WJ, B enjamin DK, Jr. Neonatal candidiasis. Infect Dis Clin North A m. 2005;19 (3):603-15.

2. Hope WW, Castagnola E, Groll AH, Roilides E, A kova M, A rendrup MC, et al. ESCM ID* guideline for the diagnosis and management of Candida diseases 2012: prevention and management of invasive infections in neonates and children caused by Candida spp. Clin M icrobiol Infect. 2012; 18 Suppl 7:38-52.

3. Kaufman DA. "Getting to Zero": preventing invasive Candida infections and eliminating infection-related mortality and morbidity in extremely preterm infants. Early Hum Dev. 2012; 88 Suppl 2:S45-9.

4. Fridkin SK, Kaufman D, Edwards JR, Shetty S, Horan T. Changing incidence of Candida bloodstream infections among NICU patients in the United States: 1995-2004. Pediatrics. 2006; 117(5):1680-7

5. Testoni D, Smith PB, Benjamin DK. The use of antifungal therapy in neonatal intensive care. Clin Perinatol. 2012; 39(1):83-98.

6. Pfaller $M, N$ eofytos $D$, Diekema $D$, Azie N, M eier-K riesche $H U$, Quan SP, et al. Epidemiology and outcomes of candidemia in 3648 patients: data from the Prospective Antifungal Therapy (PATH Alliance®) registry, 2004-2008. Diagn M icrobiol Infect Dis. 2012; 74(4):323-31.

7. M ontagna MT, Lovero G, De Giglio O, Iatta R, Caggiano G, M ontagna O, et al. Invasive fungal infections in neonatal intensive care units of Southern Italy: a multicentre regional active surveillance (AURORA project). J Prev M ed Hyg. 2010; 51(3):125-30

8. Figueras C, Heredia CD, Garcia JJ, Navarro M, Ruiz-Contreras J, Rossich R, et al. [The Spanish Society of Paediatric Infectious Diseases (SEIP) recommendations on the diagnosis and management of invasive candidiasis]. An Pediatr (B arc). 2011; 74(5):337.e1-337.e17.

9. Feja KN, Wu F, Roberts K, Loughrey M, Nesin M, Larson E, et al. Risk factors for candidemia in critically ill infants: a matched case-control study. J Pediatr. 2005; 147(2):156-61

10. Chalmers C, Gaur S, Chew J, Wright T, Kumar A, M athur S, et al. Epidemiology and management of candidaemia--a retrospective, multicentre study in five hospitals in the UK. M ycoses. 2011; 54(6):e795-800.

11. Camacho-Gonzalez A, Spearman PW, Stoll BJ. Neonatal infectious diseases: evaluation of neonatal sepsis. Pediatr Clin N orth A m. 2013; 60(2):367-89.

12. Steinbach WJ, Roilides E, Berman D, Hoffman JA, Groll AH, Bin-Hussain I, et al. Results from a prospective, international, epidemiologic study of invasive candidiasis in children and neonates. Pediatr Infect Dis ). 2012; 31(12):1252-7.

13. Chapman RL. Prevention and treatment of Candida infections in neonates. Semin Perinatol. 2007; 31(1):39-46.

14. Sardana V, Pandey A, M adan M, Goel SP, A sthana AK. Neonatal candidemia: a changing trend. Indian J Pathol M icrobiol. 2012; 55(1):132-3.

15. Benjamin DK, Stoll BJ, Gantz M G, Walsh MC, Sánchez PJ, Das A, et al. Neonatal candidiasis: epidemiology, risk factors, and clinical judgment. Pediatrics. 2010; 126(4):e865-73.

16. Oeser C, Lamagni T, Heath PT, Sharland M, Ladhani S. The epidemiology of neonatal and pediatric candidemia in England and Wales, 2000-2009. Pediatr Infect Dis J . 2013; 32(1):23-6

17. Zaoutis TE. Invasive fungal infections in pediatric patients: challenges to optimal management. J Pediatr. 2010; 156(4):A 1-S86.

18. Saiman L, Ludington E, Pfaller M, Rangel-Frausto S, Wiblin RT, Dawson J, et al. Risk factors for candidemia in $\mathrm{N}$ eonatal Intensive $\mathrm{C}$ are U nit patients. The $\mathrm{Na}$ tional Epidemiology of M ycosis Survey study group. Pediatr Infect Dis J. 2000; 19(4):319-24. 
19. Pappas PG. Invasive candidiasis. Infect Dis Clin N orth A m. 2006; 20(3):485-506.

20. Benjamin DK, Stoll BJ, Fanaroff AA, MCD onald SA, Oh W, Higgins RD, et al. Neonatal candidiasis among extremely low birth weight infants: risk factors, mortality rates, and neurodevelopmental outcomes at 18 to 22 months. Pediatrics. 2006; 117(1):84-92.

21. M iranda LN, van der Heijden IM, Costa SF, Sousa AP, Sienra RA, Gobara S et al. Candida colonisation as a source for candidaemia. J Hosp Infect. 2009; 72(1):9-16

22. B liss J M, Wong AY, B hak G, L aforce-N esbitt SS, Taylor S, Tan S, et al. Candida virulence properties and adverse clinical outcomes in neonatal candidiasis. J Pediatr. 2012; 161(3):441-7.e2.

23. Clerihew L, M cGuire W. A ntifungal therapy for newborn infants with invasive fungal infection. Cochrane Database Syst Rev. 2012; 6:CD 003953.

24. Pappas PG, K auffman CA , A ndes D, B enjamin DK, Calandra TF, Edwards JE, et al. Clinical practice guidelines for the management of candidiasis: 2009 update by the Infectious Diseases Society of A merica. Clin Infect Dis. 2009; 48(5):50335.

25. Turner K, M anzoni P, B enjamin DK, Cohen-Wolkowiez M, Smith PB, Laughon $M M$. Fluconazole pharmacokinetics and safety in premature infants. Curr M ed Chem. 2012; 19(27):4617-20

26. Wilkerson J, M CPherson C, D onze A. Fluconazole to prevent systemic fungal infections in infants: reviewing the evidence. Neonatal Netw. 2010; 29(5):323-33.

27. Egunsola $\mathrm{O}$, A defurin A, Fakis A, Jacqz-A igrain E, Choonara I, Sammons H Safety of fluconazole in paediatrics: a systematic review. Eur J Clin Pharmacol. 2013; 69(6):1211-21.

28. Stoll BJ, Hansen N, Fanaroff AA, W right LL, Carlo WA, Ehrenkranz RA, et al $L$ ate-onset sepsis in very low birth weight neonates: the experience of the NICHD Neonatal Research Network. Pediatrics 2002; 110 (2 Pt 1): 285-91.

29. Smith PB, Steinbach WJ, Cotten CM, Schell WA, Perfect JR, Walsh TJ, et al Caspofungin for the treatment of azole resistant candidemia in a premature infant. J Perinatol. 2007; 27(2):127-9.

30. Roilides E. Invasive candidiasis in neonates and children. Early Hum Dev. 2011; 87 Suppl 1:S75-6.

31. B recht $M$, Clerihew L, M cGuire W. Prevention and treatment of invasive fungal infection in very low birthweight infants. Arch Dis Child Fetal Neonatal Ed. 2009; 94(1):F65-9.

32. Hsieh E, Smith PB, Jacqz-A igrain E, K aguelidou F, Cohen-Wolkowiez M, M anzoni $P$, et al. Neonatal fungal infections: when to treat?. Early Hum Dev. 2012; 88 Suppl 2:S6-S10

33. Le J, Tran TT, Bui I, Wang M K, Vo A, A dler-Shohet FC. Time to initiation of antifungal therapy for neonatal candidiasis. A ntimicrob A gents Chemother. 2013; 57(6):2550-5

34. Greenberg RG, Benjamin DK, Gantz M G, Cotten CM, Stoll BJ, Walsh M C, et al Empiric antifungal therapy and outcomes in extremely low birth weight infants with invasive candidiasis. J Pediatr. 2012; 161(2):264-9.
35. M ohamed WA I Ismail M . A randomized, double-blind, prospective study of caspofungin vs. amphotericin B for the treatment of invasive candidiasis in newborn infants. J Trop Pediatr. 2012; 58(1):25-30.

36. Santolaya ME, Alvarado Matute T, de Queiroz Telles F, Colombo AL, Zurita J, Tiraboschi IN, et al. [Recommendations for the management of candidemia in neonates in Latin A merica. Grupo Proyecto Épico]. Rev Iberoam Micol. 2013; 30(3 Suppl 1):158-70.

37. Kaufman DA, Manzoni P. Strategies to prevent invasive candidal infection in extremely preterm infants. Clin Perinatol. 2010; 37(3):611-28.

38. El Ahmed HH, Cañadas-De la Fuente GA, Fernández-Castillo R, GonzálezJiménez $E$, Cantero-Hinojosa J, Lardón-Fernández M. [Generalized cutaneous candidiasis in newborn at term]. Biomedica. 2012; 32(2):170-3

39. Wynn JL, Tan S, Gantz M G, Das A, Goldberg RN, A dams-Chapman I, et al. Outcomes following candiduria in extremely low birth weight infants. Clin Infect Dis. 2012; 54(3):331-9.

40. Jans J, Brüggemann RJ, Christmann V, Verweij PE, Warris A. Favorable outcome of neonatal cerebrospinal fluid shunt-associated $C$ andida meningitis with caspofungin. A ntimicrob A gents Chemother. 2013;57(5):2391-3.

41. Basu S, K umar A, Kapoor K, Bagri NK, Chandra A. N eonatal endogenous endophthalmitis: a report of six cases. Pediatrics. 2013; 131(4):e1292-7.

42. Hacimustafaoglu M, Celebi S. Candida infections in non-neutropenic children after the neonatal period. Expert Rev A nti Infect Ther. 2011; 9(10):923-40.

43. Healy CM. Fungal prophylaxis in the neonatal intensive care unit. Neoreviews. 2008:9(12):e562-e70.

44. Healy CM, Campbell JR, Zaccaria E, Baker CJ. Fluconazole prophylaxis in extremely low birth weight neonates reduces invasive candidiasis mortality rates without emergence of fluconazole-resistant Candida species. Pediatrics. 2008; 121(4):703-10.

45. O'Grady MJ, Dempsey EM. Antifungal prophylaxis for the prevention of neonatal candidiasis?. A cta Paediatr. 2008: 97(4):430-3.

46. Manzoni $P$, Stolfi I, Pugni L, Decembrino L, Magnani C, Vetrano $G$, et al. A multicenter, randomized trial of prophylactic fluconazole in preterm neonates. $N$ Engl J M ed. 2007; 356(24):2483-95.

47. Cornely OA, M arty FM, Stucker F, Pappas PG, UIImann AJ. Efficacy and safety of micafungin for treatment of serious $C$ andida infections in patients with or without malignant disease. M ycoses. 2011; 54(6):e838-47.

48. Kung Y H, Hsieh Y F, Weng Y H, Lien RI, Luo J, Wang Y, et al. Risk factors of late-onset neonatal sepsis in Taiwan: A matched case-control study. J M icrobiol Immunol Infect. 2013: S1684-1182(13)00195-3.

49. Knowles SJ. Strategies for the prevention of hospital-acquired infections in the neonatal intensive care unit. J Hosp Infect. 2009; 71(1):95-6.

50. Romeo M G, Romeo DM, Trovato L, Oliveri S, Palermo F, Cota F, et al. Role of probiotics in the prevention of the enteric colonization by Candida in preterm newborns: incidence of late-onset sepsis and neurological outcome. J Perinatol. $2011 ; 31(1): 63-9$ 